\title{
Regularized Estimation of Stokes Images from Polarimetric Measurements
}

\author{
John R. Valenzuela ${ }^{a}$ and Jeffrey A. Fessler ${ }^{a}$ \\ ${ }^{a}$ University of Michigan, 1301 Beal Avenue, Ann Arbor, MI , USA 48109-2122;
}

\begin{abstract}
In the remote sensing context the goal of imaging polarimetry is to map the state of polarization of a scene of interest. The polarization state of a scene can be represented by the Stokes parameters. Since the Stokes parameters are not directly measurable one must first make several individual measurements and then the infer the Stokes parameters. We approach the Stokes parameter construction problem using penalized-likelihood estimation. Given the measured linearly polarized images, what is the optimal means by which to deblur and denoise and construct the Stokes parameters? In traditional image restoration one attempts to restore the blurred and noise corrupted data directly. In the case of imaging polarimetry we must answer the question of the optimality of restoring the measured data and then forming the Stokes images or restoring the Stokes images directly. An alternative approach is to estimate the Stokes parameters directly. We define our cost function for reconstruction by a weighted least squares data fit penalty and a regularization penalty. We show that for quadratic regularization the estimators of Stokes and intensity images can be made equal by appropriate choice of regularization parameters. It is empirically shown that, when using edge preserving regularization, estimating Stokes parameters directly leads to somewhat lower error.
\end{abstract}

Keywords: Imaging, Regularized, Polarimetric, Stokes

\section{INTRODUCTION}

\subsection{Polarimetry in Remote Sensing: Stokes Vector Imaging}

In remote sensing and astronomical applications the properties of light that are commonly measured and analyzed include intensity, wavelength, and coherence. ${ }^{1,2}$ In the context of imaging, intensity measurements provide information on scene content while spectral measurements provide additional information that can be used for material classification and target identification. A fourth property of light that is related to imaging is polarization. Polarization varies slowly with wavelength and so tends to be uncorrelated with spectral measurements ${ }^{1}$ thereby offering the potential for image enhancements not available with spectral measurements alone.

Polarization signatures are indicative of surface features of the object, i.e., surface orientation and roughness. ${ }^{1 *}$ Man made objects tend to have smoother surfaces than natural objects, so the mechanism of reflection is dominated by specular reflection which tends to retain or even enhance any polarization of the source.

The state of polarization of a transverse optical field can be represented in several ways; ${ }^{4}$ in this paper we focus on the Stokes vector representation. The Stokes vector is a four component vector, $\mathbf{S}=\left(S_{0}, S_{1}, S_{2}, S_{3}\right)$, whose elements are functions of the intensity (flux density) of the optical field. The components of the Stokes vector are defined as follows: $S_{0}$ is the total intensity, $S_{1}$ is the difference between the intensity transmitted by a linear polarizer oriented parallel to the $x\left(0^{\circ}\right.$ reference) axis and one oriented parallel to the $y$ axis, $S_{2}$ is the difference between the intensity transmitted by a linear polarizer oriented at $45^{\circ}$ to the $x$ axis and one oriented at $135^{\circ}$, and $S_{3}$ is the difference between the intensity transmitted by a right circular polarizer and a left circular polarizer. In the vast majority of remote sensing applications the component $S_{3}$ is negligible, for this reason it is typical to work with only the first three components of the Stokes vector.

The intensity passed by a linear polarizer, whose transmission axis is oriented at angle $\theta$, may be written in terms of the components of the Stokes vector. The effect of an ideal linear polarizer is to pass that part of the

* In general, polarization signatures can give information about material composition however those applications reside largely in the field of ellipsometry ${ }^{3}$ and are outside the scope of this work.

Computational Imaging VI, edited by Charles A. Bouman, Eric L. Miller, llya Pollak,

Proc. of SPIE-IS\&T Electronic Imaging, SPIE Vol. 6814, 681403, @ 2008 SPIE-IS\&T · 0277-786X/08/\$18 
electric field which is along the transmission axis of the polarizer. Let the transmission axis of the polarizer be $\mathbf{p}_{\theta}=\cos (\theta) \hat{\mathbf{i}}+\sin (\theta) \hat{\mathbf{j}}$ and the electric field be

$$
\mathbf{E}(t)=E_{x}(t) \hat{\mathbf{i}}+E_{y}(t) \hat{\mathbf{j}}
$$

The intensity, $\Gamma(\theta)$, passed by the polarizer is then ${ }^{\dagger}$

$$
\begin{aligned}
\Gamma(\theta) & =\left\langle\left(\mathbf{E}(t) \cdot \mathbf{p}_{\theta}\right)^{2}\right\rangle \\
& =\left\langle E_{x}^{2}(t)\right\rangle \cos ^{2}(\theta)+\left\langle E_{y}^{2}(t)\right\rangle \sin ^{2}(\theta)+2\left\langle E_{x}(t) E_{y}(t)\right\rangle \sin (\theta) \cos (\theta) .
\end{aligned}
$$

Using of the identities $\cos ^{2}(\theta)=(1+\cos (2 \theta)) / 2$ and $\sin ^{2}(\theta)=(1-\cos (2 \theta)) / 2$ we can rewrite the expression for the intensity as

$$
\Gamma(\theta)=\frac{1}{2}\left(\left\langle E_{x}^{2}(t)\right\rangle+\left\langle E_{y}^{2}(t)\right\rangle+\left(\left\langle E_{x}^{2}(t)\right\rangle-\left\langle E_{y}^{2}(t)\right\rangle\right) \cos (2 \theta)+2\left\langle E_{x}(t) E_{y}(t)\right\rangle \sin (2 \theta)\right) .
$$

The components of the Stokes vector are defined in terms of optical intensity, and can be written in terms of the electric field, as follows

$$
\begin{aligned}
& S_{0}=\Gamma\left(0^{\circ}\right)+\Gamma\left(90^{\circ}\right)=\left\langle E_{x}^{2}(t)\right\rangle+\left\langle E_{y}^{2}(t)\right\rangle \\
& S_{1}=\Gamma\left(0^{\circ}\right)-\Gamma\left(90^{\circ}\right)=\left\langle E_{x}^{2}(t)\right\rangle-\left\langle E_{y}^{2}(t)\right\rangle \\
& S_{2}=\Gamma\left(45^{\circ}\right)-\Gamma\left(135^{\circ}\right)=2\left\langle E_{x}(t) E_{y}(t)\right\rangle,
\end{aligned}
$$

and, by direct substitution, the intensity is related to the Stokes parameters as follows

$$
\Gamma(\theta)=\frac{1}{2}\left(S_{0}+S_{1} \cos (2 \theta)+S_{2} \sin (2 \theta)\right) .
$$

For $J$ measurement angles $\left\{\theta_{1}, \ldots, \theta_{J}\right\}$ equation (7) becomes a system of $J$ equations. In matrix form the system is

$$
\Gamma=\mathbf{T}_{J \times 3} \mathbf{S} .
$$

where $\boldsymbol{\Gamma}=\left(\left(\Gamma\left(\theta_{1}\right), \ldots, \Gamma\left(\theta_{J}\right)\right)\right.$ and $\mathbf{S}=\left(S_{0}, S_{1}, S_{2}\right)$. The conventional estimate of the Stokes vector uses the pseudoinverse $^{\ddagger}$ (if it exists) of $\mathbf{T}_{J \times 3}$ :

$$
\hat{\mathbf{S}}=\mathbf{T}_{J \times 3}^{\dagger} \boldsymbol{\Gamma} .
$$

Moreover, note that the model (7) and the estimate (9) have ignored noise, blur, and other degradations.

\section{IMAGE RECONSTRUCTION APPLIED TO STOKES VECTOR IMAGING}

Traditionally, statistical image reconstruction techniques are applied to data that has been corrupted by nonideal system effects, i.e., noise and blur. When applying a reconstruction algorithm to polarimetric imagery we are confronted with the question of which image set to reconstruct: the polarimetric intensity images or the underlying Stokes images. While access to the Stokes images is the ultimate goal we must be concerned with the low signal levels in the $S_{1}$ and $S_{2}$ images. On the other hand, the intensity images do not have this low signal difficulty and so are good candidates for improvement through image reconstruction. We investigate both approaches theoretically and numerically. Estimation of the intensity images is referred to as the traditional approach and estimation of the Stokes vector is referred to as the proposed approach. We explore both a quadratically penalized weighted least squares estimator and a weighted least squares estimator with edgepreserving regularization.

\footnotetext{
$\dagger\langle\cdot\rangle$ indicates time averaging.

$\ddagger$ The pseudoinverse will be indicated by a superscript $\dagger$.
} 


\subsection{Traditional Image Restoration Approach}

In the traditional approach to image restoration we try to recover the uncorrupted images from the noisy images individually. For the polarimetric imaging problem this translates into first restoring the true intensity images, and then converting those images into Stokes space via the linear transformation (9). We treat the general case of $J$ polarimetric channels (images) each having a unique polarization angle associated with it.

Denote the lexicographically ordered data of the $j$ th channel by $y_{j}$, the system matrix, that represents physical effects such as optical and detector blur, by $A_{j}$, and the noise vector by $\varepsilon_{j}$. The data vectors $\left\{y_{j}\right\}$ are each of length $n_{d}$. The size of the system matrix of the $j$ th channel is $n_{d} \times n_{p}$ where $n_{p}$ is the number of pixels in an individual true intensity image $\Gamma_{j}\left(\operatorname{In}\right.$ general, $\left.n_{d} \neq n_{p}\right)$. The noise vector, $\varepsilon_{j}$, for the $j$ th channel is of length $n_{d}$. The model for the $j$ th channel of the collected data is

$$
y_{j}=A_{j} \Gamma_{j}+\varepsilon_{j} .
$$

For simplicity, we adopt a zero-mean iid Gaussian noise model:

$$
\varepsilon_{j} \sim N\left(\mathbf{0}, \sigma^{2} \mathbf{I}\right) .
$$

The likelihood function of the $j$ th data channel, $y_{j}$, given the intensity image $\Gamma_{j}$

$$
p\left(y_{j} \mid \Gamma_{j}\right)=\frac{1}{\sqrt{\sigma^{2}(2 \pi)^{n_{d}}}} \exp \left(-\frac{1}{2 \sigma^{2}}\left(y_{j}-A_{j} \Gamma_{j}\right)^{\prime}\left(y_{j}-A_{j} \Gamma_{j}\right)\right)
$$

and the total likelihood function for the data set can then be written

$$
p(\mathbf{y} \mid \boldsymbol{\Gamma})=\frac{1}{\sqrt{\sigma^{2}(2 \pi)^{N_{d}}}} \exp \left(-\frac{1}{2 \sigma^{2}}(\mathbf{y}-\mathbf{A} \boldsymbol{\Gamma})^{\prime}(\mathbf{y}-\mathbf{A} \boldsymbol{\Gamma})\right)
$$

where $N_{d}=J n_{d}$ is the total number of measured pixels and

$$
\mathbf{y}=\left(y_{1}, \ldots, y_{J}\right), \quad \boldsymbol{\Gamma}=\left(\Gamma_{1}, \ldots, \Gamma_{J}\right), \quad \mathbf{A}=\operatorname{diag}\left(A_{j}, \ldots, A_{J}\right) .
$$

A penalized-likelihood estimator for $\boldsymbol{\Gamma}$ is given by

$$
\hat{\boldsymbol{\Gamma}}=\min _{\boldsymbol{\Gamma}}\left\{-\log p(\mathbf{y} \mid \boldsymbol{\Gamma})+R_{\Gamma}(\boldsymbol{\Gamma})\right\}
$$

where $R_{\Gamma}$ is a regularization function for the intensity images. Typically, $R_{\Gamma}(\boldsymbol{\Gamma})$ separates, i.e.,

$$
R_{\Gamma}(\boldsymbol{\Gamma})=\sum_{j=1}^{J} R_{j}\left(\Gamma_{j}\right),
$$

and the likelihood also separates, so the minimization problem for $\hat{\boldsymbol{\Gamma}}$ separates into $J$ individual regularized image restoration operations.

The estimated Stokes vector, $\hat{\mathbf{S}}_{\Gamma}$, is then calculated by using a generalized version of equation (9) that is appropriate for images. The generalization to images is accomplished by writing the transformation matrix so that we have a pixel-by-pixel version of equation (9), define $\check{\mathbf{T}}=\mathbf{T}_{J \times 3} \otimes \mathbf{I}_{n_{p} \times n_{p}}$ so that ${ }^{\S}$

$$
\hat{\mathbf{S}}_{\Gamma}=\check{\mathbf{T}}^{\dagger} \boldsymbol{\Gamma}
$$

\footnotetext{
${ }^{\S} \mathbf{I}_{n_{p} \times n_{p}}$ is the $n_{p} \times n_{p}$ identity matrix and $\otimes$ denotes Kronecker product.
} 


\subsection{Stokes Estimation Model}

The model for estimating the Stokes vector (images) differs from from the traditional estimation model in both the likelihood function and the regularization function. The likelihood function, under the same system and noise model is

$$
p(\mathbf{y} \mid \mathbf{S})=\frac{1}{\sqrt{\sigma^{2}(2 \pi)^{N_{d}}}} \exp \left(-\frac{1}{2 \sigma^{2}}(\mathbf{y}-\mathbf{A} \check{\mathbf{T}} \mathbf{S})^{\prime}(\mathbf{y}-\mathbf{A} \check{\mathbf{T}} \mathbf{S})\right) .
$$

A penalized-likelihood estimator for the Stokes vector is

$$
\hat{\mathbf{S}}=\min _{\mathbf{S}}\left\{-\log p(\mathbf{y} \mid \mathbf{S})+R_{S}(\mathbf{S})\right\}
$$

where $R_{S}$ is a regularization function for the Stokes images. As in the traditional estimator the regularization function $R_{S}$ typically separates, i.e.,

$$
R_{S}(\mathbf{S})=\sum_{j=1}^{3} R_{j}\left(S_{j}\right) .
$$

However, unlike the traditional case the likelihood function $p(\mathbf{y} \mid \mathbf{S})$ does not separate and so the minimization problem is coupled.

\section{ANALYTICAL ESTIMATOR ANALYSIS: QUADRATICALLY PENALIZED WEIGHTED LEAST SQUARES ESTIMATOR}

This section compares analytically the traditional and Stokes estimators. The degrading effect of the imaging system is taken to be optical blur and represented by the matrix $B$, i.e., $A_{j}=B$ in equation (10). This matrix is Toeplitz if the system is shift invariant, but the analysis that follows applies to a general $B$ matrix. We emphasize that each channel has the same optical and detector blur, this is a reasonable assumption because aberrations and detector effects are not affected by linear polarizers.

\subsection{Traditional Estimator}

We focus on the case of four polarimetric measurements (taken with linear polarizers) at angles $\left[0^{\circ}, 45^{\circ}, 90^{\circ}, 135^{\circ}\right]$; this sets the size of $\mathbf{T}_{J \times 3}$ in equation (8) to $\mathbf{T}_{4 \times 3}$. The Stokes to intensity transformation, $\mathbf{T}_{4 \times 3}$, and the intensity to Stokes transformation, $\mathbf{T}_{4 \times 3}^{\dagger}$, are given by

$$
\mathbf{T}_{4 \times 3}=\left[\begin{array}{ccc}
\frac{1}{2} & \frac{1}{2} & 0 \\
\frac{1}{2} & 0 & \frac{1}{2} \\
\frac{1}{2} & -\frac{1}{2} & 0 \\
\frac{1}{2} & 0 & -\frac{1}{2}
\end{array}\right] \text { and } \mathbf{T}_{4 \times 3}^{\dagger}=\left[\begin{array}{cccc}
\frac{1}{2} & \frac{1}{2} & \frac{1}{2} & \frac{1}{2} \\
1 & 0 & -1 & 0 \\
0 & 1 & 0 & -1
\end{array}\right] \text {. }
$$

The data model is

$$
\mathbf{y}=\left(\mathbf{I}_{4 \times 4} \otimes B\right) \boldsymbol{\Gamma}+\varepsilon,
$$

and the quadratic regularizing penalty function is formed using vertical and horizontal neighboring pixels. In one dimension the regularizer, $R_{\Gamma}(\Gamma)$, is written

$$
\begin{aligned}
R_{\Gamma}(\Gamma) & =\frac{1}{2} \beta \sum_{j=1}^{J} \sum_{k=2}^{n_{p}}\left(\Gamma_{j_{k}}-\Gamma_{j_{k-1}}\right)^{2} \\
& =\frac{1}{2} \beta \sum_{j=1}^{J}\left\|C \Gamma_{j}\right\|^{2}
\end{aligned}
$$


where $C$ is a finite differencing matrix and $\beta$ is the regularization "tuning" parameter. ${ }^{\text {In }}$ In two dimensions the summation notation becomes cumbersome and so we use linear algebra notation exclusively. Let $C$ be a finite differencing matrix that takes both vertical and horizontal differences, then the regularization function can be succinctly written, noting that $\mathbf{C} \stackrel{\text { def }}{=} \mathbf{I}_{4 \times 4} \otimes C$, as

$$
R_{\Gamma}(\boldsymbol{\Gamma})=\frac{1}{2} \beta\|\mathbf{C} \boldsymbol{\Gamma}\|^{2} .
$$

The total cost function can then be written

$$
\hat{\boldsymbol{\Gamma}}=\min _{\boldsymbol{\Gamma}}\left\{\frac{1}{2 \sigma^{2}}\left\|\mathbf{y}-\left(\mathbf{I}_{4 \times 4} \otimes B\right) \boldsymbol{\Gamma}\right\|^{2}+\frac{1}{2} \beta\|\mathbf{C} \boldsymbol{\Gamma}\|^{2}\right\} .
$$

We consider the case of unconstrained optimization and so the minimizer must satisfy

$$
\nabla_{\boldsymbol{\Gamma}}\left(\frac{1}{2 \sigma^{2}}\left\|\mathbf{y}-\left(\mathbf{I}_{4 \times 4} \otimes B\right) \boldsymbol{\Gamma}\right\|^{2}+\frac{1}{2} \beta\|\mathbf{C} \boldsymbol{\Gamma}\|^{2}\right)=\mathbf{0} .
$$

We can solve the above equation for $\hat{\boldsymbol{\Gamma}}$ yielding

$$
\begin{aligned}
\hat{\boldsymbol{\Gamma}} & =\left(\mathbf{I}_{4 \times 4} \otimes\left[B^{\prime} B+\sigma^{2} \beta R\right]^{-1} B^{\prime}\right) \mathbf{y} \\
\hat{\mathbf{S}}_{\Gamma} & =\check{\mathbf{T}}^{\dagger} \hat{\boldsymbol{\Gamma}},
\end{aligned}
$$

where $R=C^{\prime} C$.

\subsection{Stokes Estimator}

The data model for Stokes estimation is written slightly different than the traditional estimation approach, noting that the system effects are identical to the case of the traditional estimator we write

$$
\mathbf{y}=\left(\mathbf{T}_{4 \times 3} \otimes B\right) \mathbf{S}+\varepsilon .
$$

Following the same procedure as in the case of the traditional estimator we have the following implicitly defined estimator

$$
\hat{\mathbf{S}}=\min _{\mathbf{S}}\left\{\frac{1}{2 \sigma^{2}}\left\|\mathbf{y}-\left(\mathbf{T}_{4 \times 3} \otimes B\right) \mathbf{S}\right\|^{2}+\frac{1}{2}\left(\beta_{0}\left\|C S_{0}\right\|^{2}+\beta_{1}\left\|C S_{1}\right\|^{2}+\beta_{2}\left\|C S_{2}\right\|^{2}\right)\right\} .
$$

We remark that in this case the images being estimated are very different and so justify three independent regularization "tuning" parameters. The minimizer in this case must satisfy the condition

$$
\nabla_{\mathbf{S}}\left\{\frac{1}{2 \sigma^{2}}\left\|\mathbf{y}-\left(\mathbf{T}_{4 \times 3} \otimes B\right) \mathbf{S}\right\|^{2}+\frac{1}{2}\left(\beta_{0}\left\|C S_{0}\right\|^{2}+\beta_{1}\left\|C S_{1}\right\|^{2}+\beta_{2}\left\|C S_{2}\right\|^{2}\right)\right\}=\mathbf{0},
$$

which leads to the closed form estimator

$$
\hat{\mathbf{S}}=\left[\mathbf{T}_{4 \times 3}^{\prime} \mathbf{T}_{4 \times 3} \otimes B^{\prime} B+\sigma^{2} \beta_{3 \times 3} \otimes R\right]^{-1}\left(\mathbf{T}_{4 \times 3}^{\prime} \otimes B^{\prime}\right) \mathbf{y}
$$

where $\beta_{3 \times 3}=\operatorname{diag}\left(\beta_{0}, \beta_{1}, \beta_{2}\right)$, and $R=C^{\prime} C$.

\footnotetext{
IThe regularization parameter is not expected differ between the different polarimetric channels as there is a high correlation between them.
} 


\subsection{Spatial Resolution Analysis of the Stokes Estimator}

We begin by calculating the mean value of the $\hat{\mathbf{S}}$ estimator. To aid in the calculation define $P=\operatorname{diag}(1,2,2)$, $P^{-1}=\operatorname{diag}\left(1, \frac{1}{2}, \frac{1}{2}\right)$. Then

$$
\begin{aligned}
E[\hat{\mathbf{S}} \mid \mathbf{S}] & =\left[\mathbf{T}_{4 \times 3}^{\prime} \mathbf{T}_{4 \times 3} \otimes B^{\prime} B+\sigma^{2} \beta_{3 \times 3} \otimes R\right]^{-1}\left(\mathbf{I}_{3 \times 3} \otimes B^{\prime}\right)\left(P^{-1} \otimes \mathbf{I}_{n_{p} \times n_{p}}\right)\left(\mathbf{T}_{4 \times 3}^{\dagger} \otimes \mathbf{I}_{n_{p} \times n_{p}}\right) E[\mathbf{y} \mid \mathbf{S}] \\
& =\left[\mathbf{T}_{4 \times 3}^{\prime} \mathbf{T}_{4 \times 3} \otimes B^{\prime} B+\sigma^{2} \beta_{3 \times 3} \otimes R\right]^{-1}\left(\mathbf{I}_{3 \times 3} \otimes B^{\prime}\right)\left(P^{-1} \otimes \mathbf{I}_{n_{p} \times n_{p}}\right)\left(\mathbf{T}_{4 \times 3}^{\dagger} \otimes \mathbf{I}_{n_{p} \times n_{p}}\right)\left(\mathbf{T}_{4 \times 3} \otimes B\right) \mathbf{S} \\
& =\left[\mathbf{T}_{4 \times 3}^{\prime} \mathbf{T}_{4 \times 3} \otimes B^{\prime} B+\sigma^{2} \beta_{3 \times 3} \otimes R\right]^{-1}\left(\mathbf{I}_{3 \times 3} \otimes B^{\prime}\right)\left(P^{-1} \otimes \mathbf{I}_{n_{p} \times n_{p}}\right)\left(\mathbf{I}_{3 \times 3} \otimes B\right) \mathbf{S} \\
& =\underbrace{\left[P^{-1} \otimes B^{\prime} B+\sigma^{2} \beta_{3 \times 3} \otimes R\right]^{-1}\left(P^{-1} \otimes B^{\prime} B\right)}_{L} \mathbf{S} \\
& =L \mathbf{S},
\end{aligned}
$$

where $L$ is a $\left[3 n_{p} \times 3 n_{p}\right]$ matrix that acts somewhat like a Wiener filter. Each term in the above expression is uncoupled since the matrices $P^{-1}$ and $\beta_{3 \times 3}$ are diagonal. To explore the spatial resolution properties of the estimators, we approximate $B^{\prime} B$ and $R$ by circulant matrices. Let the eigen-decompostions of $B^{\prime} B$ and $R$ be approximated by $Q \Theta^{\prime} \Theta Q^{\prime}$ and $Q \Omega Q^{\prime}$ respectivelyll. Then

$$
\begin{aligned}
E[\hat{\mathbf{S}} \mid \mathbf{S}] & =\left[P^{-1} \otimes Q \Theta^{\prime} \Theta Q^{\prime}+\sigma^{2} \beta_{3 \times 3} \otimes Q \Omega Q^{\prime}\right]^{-1}\left(P^{-1} \otimes Q \Theta^{\prime} \Theta Q^{\prime}\right) \mathbf{S} \\
& =Q\left\{\left[P^{-1} \otimes \Theta^{\prime} \Theta+\sigma^{2} \beta_{3 \times 3} \otimes \Omega\right]^{-1}\left(P^{-1} \otimes \Theta^{\prime} \Theta\right)\right\} Q^{\prime} \mathbf{S} .
\end{aligned}
$$

We see that the expectation of $\hat{\mathbf{S}}$ is approximately a filtered version of the true objects with each image having an independent filter.

$$
\begin{aligned}
& \text { Filter for } S_{0} \quad \Longrightarrow \quad L_{k}=\frac{\left|B_{k}\right|^{2}}{\left|B_{k}\right|^{2}+\beta_{0} \sigma^{2} R_{k}} \\
& \text { Filter for } S_{1} \quad \Longrightarrow \quad L_{k}=\frac{\frac{1}{2}\left|B_{k}\right|^{2}}{\frac{1}{2}\left|B_{k}\right|^{2}+\beta_{1} \sigma^{2} R_{k}} \\
& \text { Filter for } S_{2} \quad \Longrightarrow \quad L_{k}=\frac{\frac{1}{2}\left|B_{k}\right|^{2}}{\frac{1}{2}\left|B_{k}\right|^{2}+\beta_{2} \sigma^{2} R_{k}},
\end{aligned}
$$

$k=1, \ldots, n_{p}$. We see that the $S_{1}$ and $S_{2}$ channels have a different spatial resolution than the $S_{0}$ channel, unless we choose $\beta_{1}=\beta_{2}=\beta_{0} / 2$. However, in the intensity model all three channels always have identical resolutions. Matching the spatial resolution of the $S_{1}$ and $S_{2}$ channels to the $S_{0}$ channel also leads to the uncoupling of estimator. That is, by choosing $\beta_{3 \times 3}=\beta \operatorname{diag}\left(1, \frac{1}{2}, \frac{1}{2}\right)$ we have

$$
\begin{aligned}
\hat{\mathbf{S}} & =\left[\mathbf{T}_{4 \times 3}^{\prime} \mathbf{T}_{4 \times 3} \otimes B^{\prime} B+\sigma^{2} \beta_{3 \times 3} \otimes R\right]^{-1}\left(\mathbf{T}_{4 \times 3}^{\prime} \otimes B^{\prime}\right) \mathbf{y} \\
& =\left[\operatorname{diag}\left(1, \frac{1}{2}, \frac{1}{2}\right) \otimes\left(B^{\prime} B+\sigma^{2} \beta R\right)\right]^{-1}\left(\mathbf{T}_{4 \times 3}^{\prime} \otimes B^{\prime}\right) \mathbf{y} \\
& =\operatorname{diag}(1,2,2) \otimes\left[B^{\prime} B+\sigma^{2} \beta R\right]^{-1}\left(\mathbf{T}_{4 \times 3}^{\prime} \otimes B^{\prime}\right) \mathbf{y} \\
& =\mathbf{T}_{4 \times 3}^{\dagger} \otimes\left[B^{\prime} B+\sigma^{2} \beta R\right]^{-1} B^{\prime} \mathbf{y} \\
& =\hat{\mathbf{S}}_{\Gamma} .
\end{aligned}
$$

The Stokes estimator has uncoupled and reduced to the traditional estimator.

\footnotetext{
"Q denotes the orthonormal DFT matrix.
} 


\subsection{Edge-preserving Regularization}

In the Stokes images $\left\{S_{1}, S_{2}\right\}$ the polarization information typically has sharp edges. To recover as much polarization information as possible the regularization function should preserve edges as much as possible. Since the quadratic regularization tends to wash out edges and smooth noise we explore edge preserving regularization using the hyperbolic function $\psi(t ; \delta)=\delta^{2}\left(\sqrt{1+\left(\frac{t}{\delta}\right)^{2}}-1\right)$. For fixed $\delta$ this function is approximately quadratic for values of $t<\delta$ and approximately linear for $t>\delta$. This behavior will tend to smooth noise and preserve edges. The Stokes and intensity estimators now written

$$
\begin{aligned}
& \hat{\mathbf{S}}=\min _{\mathbf{S}}\left\{\frac{1}{2 \sigma^{2}}\left\|\mathbf{y}-\left(\mathbf{T}_{4 \times 3} \otimes B\right) \mathbf{S}\right\|^{2}+\left(\beta_{0} \psi\left(C S_{0} ; \delta_{0}\right)+\beta_{1} \psi\left(C S_{1} ; \delta_{1}\right)+\beta_{2} \psi\left(C S_{2} ; \delta_{2}\right)\right)\right\} \\
& \hat{\boldsymbol{\Gamma}}=\min _{\boldsymbol{\Gamma}}\left\{\frac{1}{2 \sigma^{2}}\left\|\mathbf{y}-\left(\mathbf{I}_{4 \times 4} \otimes B\right) \mathbf{\Gamma}\right\|^{2}+\beta \sum_{j} \psi\left(C \Gamma_{j} ; \delta\right)\right\}
\end{aligned}
$$

The Stokes estimator now has two regularization parameters per Stokes image and the intensity estimator has two regularization parameters in total**. Since closed form expressions for the minimizers of these cost functions are intractable we minimize (36) and (37) numerically. The numerical optimization was done with the LBFGS algorithm. ${ }^{5}$ To obtain optimal values of all regularization parameter combinations would be computationally burdensome so we chose the $\beta$ parameters by analyzing the local point spread function of the quadratic estimators. The $\beta$ parameters are chosen so that the FWHM of the estimator is a prescribed amount in regions where the regularization function is approximately quadratic. The local impulse response is defined by

$$
l_{j}(\mathbf{S})=\lim _{\varepsilon \rightarrow 0} \frac{\hat{\mathbf{S}}\left(\bar{y}\left(\mathbf{S}+\varepsilon \mathbf{e}_{j}\right)-\hat{\mathbf{S}}(\bar{y}(\mathbf{S}))\right.}{\varepsilon}=\nabla \hat{\mathbf{S}}(\bar{y}(\mathbf{S})) \nabla \bar{y}(\mathbf{S}) \mathbf{e}_{j} .
$$

For the quadratically penalized weighted least squares estimator with white Gaussian noise the local impulse response of each Stokes image is independent and written ${ }^{6}$

$$
l_{k_{j}}=\left[B^{\prime} B+\sigma^{2} \beta_{k} R\right]^{-1} B^{\prime} B \mathbf{e}_{k_{j}} \quad \text { where } k \in\{0,1,2\} \text { indicates the Stokes image. }
$$

Using a local Fourier approximation we can compute this impulse response with FFTs. Since the FFTs are computationally inexpensive we can sweep over the $\beta$ parameters and choose the one that corresponds to an a priori FWHM of the impulse response. By choosing $\beta$ so that the estimator has controlled noise smoothing properties we can vary the $\delta$ parameters to find optimal values.

\section{SIMULATION EXPERIMENTS}

Simulation experiments were performed to evaluate the estimators (36) and (37). For the true imagery we used polarimetric images that were collected by General Dynamics Advanced Information Systems in Ypsilanti, Michigan; the polarization angles of the sensor were $\left\{0^{\circ}, 45^{\circ}, 90^{\circ}, 135^{\circ}\right\}$. We added known blur and Gaussian noise to the imagery. The system PSF was had a FWHM of 1.9 pixels; the PSF was constructed from a phase screen, parameterized by a uniform distribution of the first 20 Zernike polynomials, placed in an annular pupil. The SNR of data was $25 \mathrm{~dB} .{ }^{\dagger \dagger}$ The noisy and blurred polarimetric imagery is shown in Figure 1.

The regularization parameters $\left[\beta_{0}, \beta_{1}, \beta_{2}, \beta\right]$ were chosen so that the FWHM of each estimator PSF was 1.5 pixels and the spatial resolution across the Stokes channels was constant (recall equation (34)). The second set of regularization parameters, $\left[\delta_{0}, \delta_{1}, \delta_{2}, \delta\right]$, was determined by sweeping over a range and choosing the parameters which yielded a minimum RMS error in the estimate.

\footnotetext{
${ }^{* *}$ Recall that the intensity images are highly correlated and so we do not expect them to have different regularization parameters.

${ }^{\dagger \dagger}$ We define $\mathrm{SNRdB}=20 \log _{10}(\|\bar{y}\| /\|\bar{y}-y\|)$ where $\bar{y}$ and $y$ are the uncorrupted and corrupted images respectively.
} 


\section{RESULTS}

Table 1 compares the RMS errors of four quantities: (1) The total Stokes estimate, (2) the $S_{0}$ estimate, (3) the $S_{1}$ estimate, the $S_{2}$ estimate, and (4) the estimate of the degree of linear polarization (DOLP). The DOLP is a useful quantity in polarimetric image analysis and is defined by DOLP $=\sqrt{S_{1}^{2}+S_{2}^{2}} / S_{0}$.

The noisy and blurred data is shown in Figure 1, and the estimated polarimetric imagery using the traditional estimation method is shown in Figure 2. Comparison of the pristine imagery and estimates for $S_{0}$ are shown in Figure 3, estimates of $S_{1}$ are shown in Figure 4, estimates of $S_{2}$ are shown in Figure 5, and estimates of DOLP are shown in Figure 6.

\section{CONCLUSIONS AND FUTURE WORK}

Estimation of Stokes vectors directly provides estimates with modestly lower overall RMS error as compared with restoring the intensity images and then transforming to Stokes space for interpretation. Future work includes further investigation into regularization optimization and addressing non idealities such aliasing and broadband PSF effects. Also, investigating estimator efficiency and convergence properties will be done.

\section{ACKNOWLEDGMENTS}

The first author thanks General Dynamics Advanced Imaging Systems for providing polarimetric data and the LBFGS code and Brendhan Givens (GD-AIS) for his guidance and mentoring.

\section{REFERENCES}

1. J. Scott Tyo, Dennis L. Goldstein, David B. Chenault, and Joseph A. Shaw, "Review of passive imaging polarimetry for remote sensing applications", Applied Optics 45 (22) (2006)

2. Harrison H. Barrett and Kyle J. Myers, Foundations of Image Science (John Wiley and Sons, Inc., 2004)

3. R.M.A. Azzam and N.M. Bashara, Ellipsometry and Polarized Light (Elsevier Science Publishers B.V., 1987)

4. R.A. Chipman, "Polarimetry", in Handbook of Optics (McGraw-Hill, 1995), Vol 2, Chap. 22.

5. D. Liu, J. Nocedal, "On the limited memory BFGS method for large scale optimization", Mathematical Programming, 45(3), pp. 503-528 (1989)

6. J.A. Fessler and W.L. Rogers, "Spatial resolution properties of penalized-likelihood image reconstruction methods: Space-invariant tomographs" in IEEE Trans. Image Processing 5 pp. 1346-58 Sept. 1996

Table 1. Simulation Results

\begin{tabular}{|c|c|c|}
\hline & Stokes Estimator Fractional RMSE & Traditional Estimator Fractional RMSE \\
\hline$\hat{S}_{0}$ & $1.33 \%$ & $1.30 \%$ \\
\hline$\hat{S}_{1}$ & $18.5 \%$ & $27.9 \%$ \\
\hline$\hat{S}_{2}$ & $25.5 \%$ & $38.1 \%$ \\
\hline DOLP & $21.5 \%$ & $31.0 \%$ \\
\hline
\end{tabular}



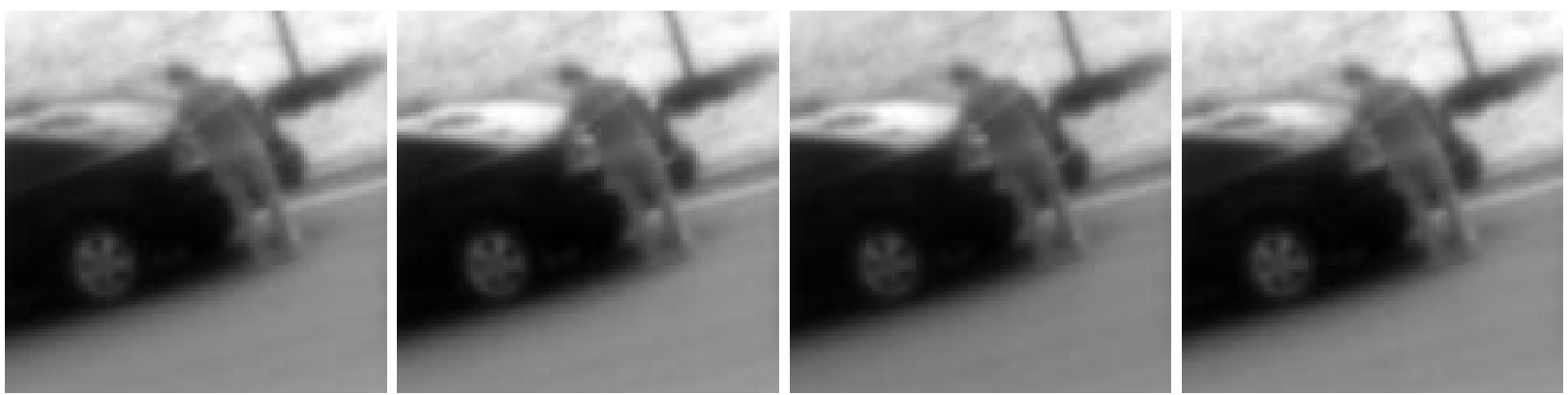

Figure 1. Noisy and blurred polarimetric imagery. From left to right the angle of the polarizer is $\left\{0^{\circ}, 45^{\circ}, 90^{\circ}, 135^{\circ}\right\}$
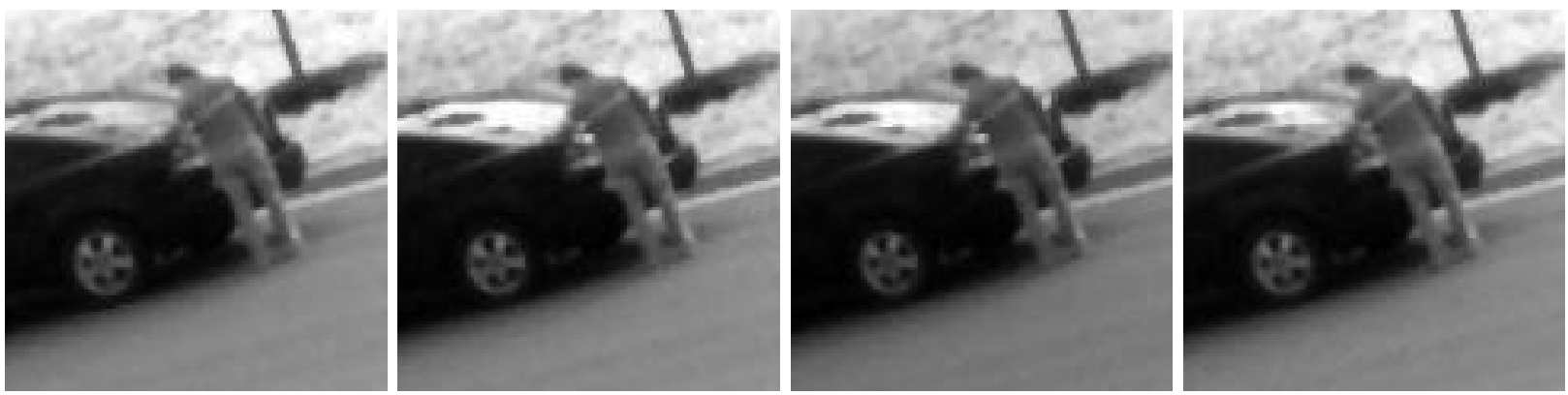

Figure 2. Estimated polarimetric imagery. From left to right the angle of the polarizer is $\left\{0^{\circ}, 45^{\circ}, 90^{\circ}, 135^{\circ}\right\}$
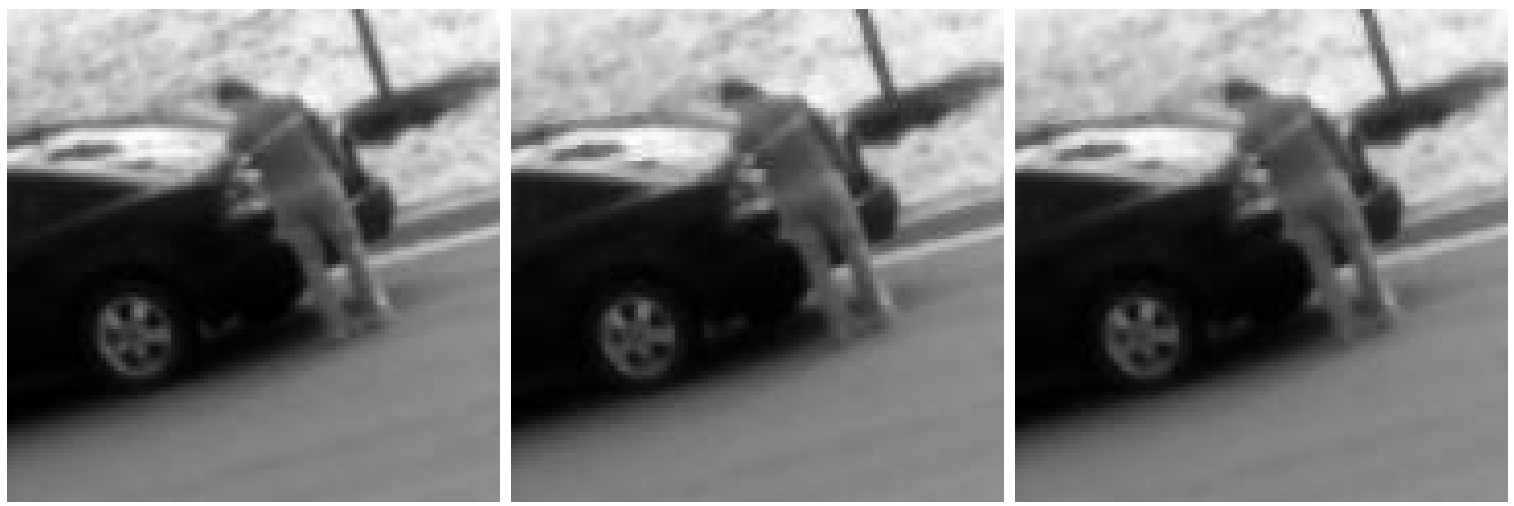

Figure 3. Estimates of $S_{0}$. From left to right: Pristine, Proposed Method, Traditional Method
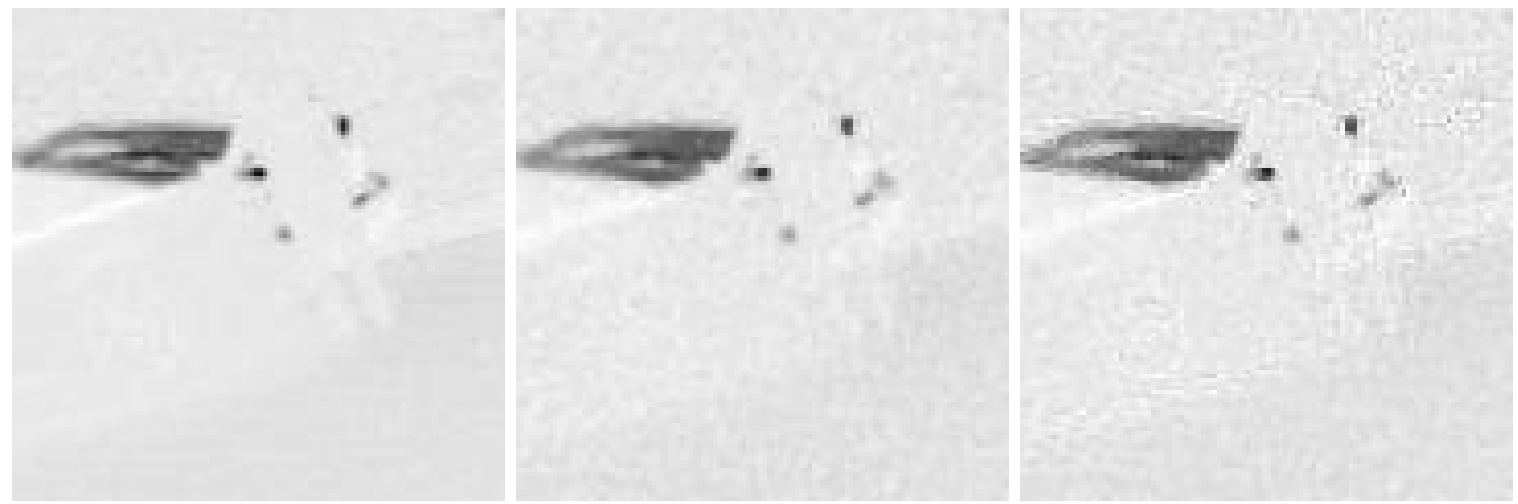

Figure 4. Estimates of $S_{1}$. From left to right: Pristine, Proposed Method, Traditional Method 

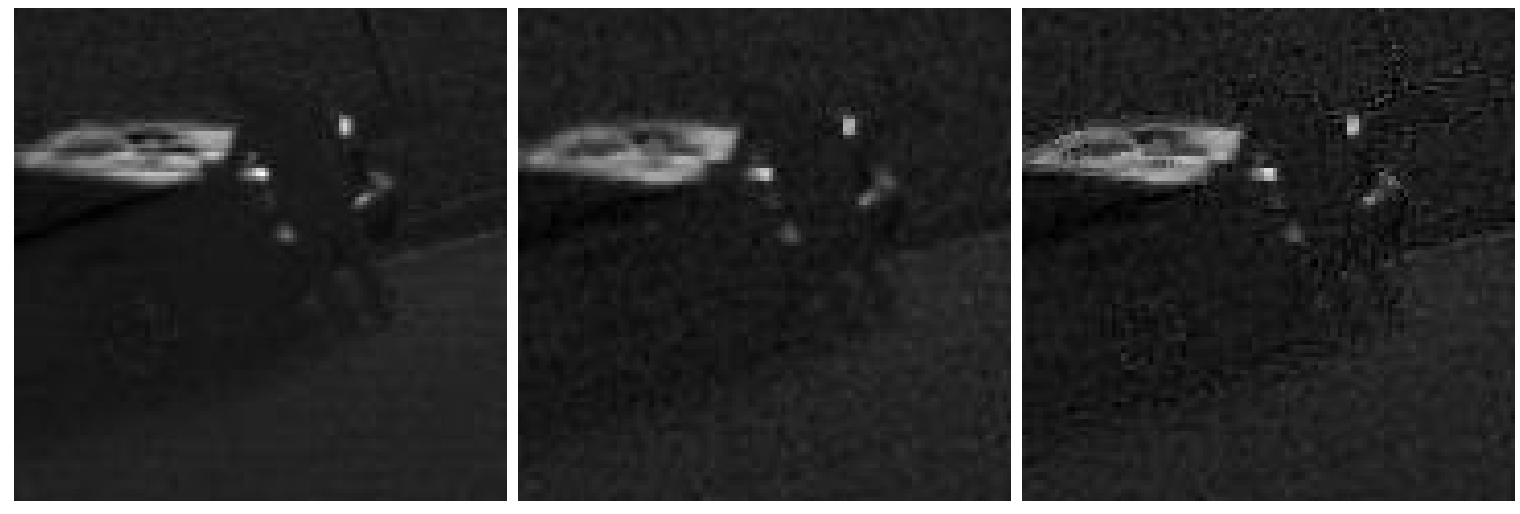

Figure 5. Estimates of $S_{2}$. From left to right: Pristine, Proposed Method, Traditional Method
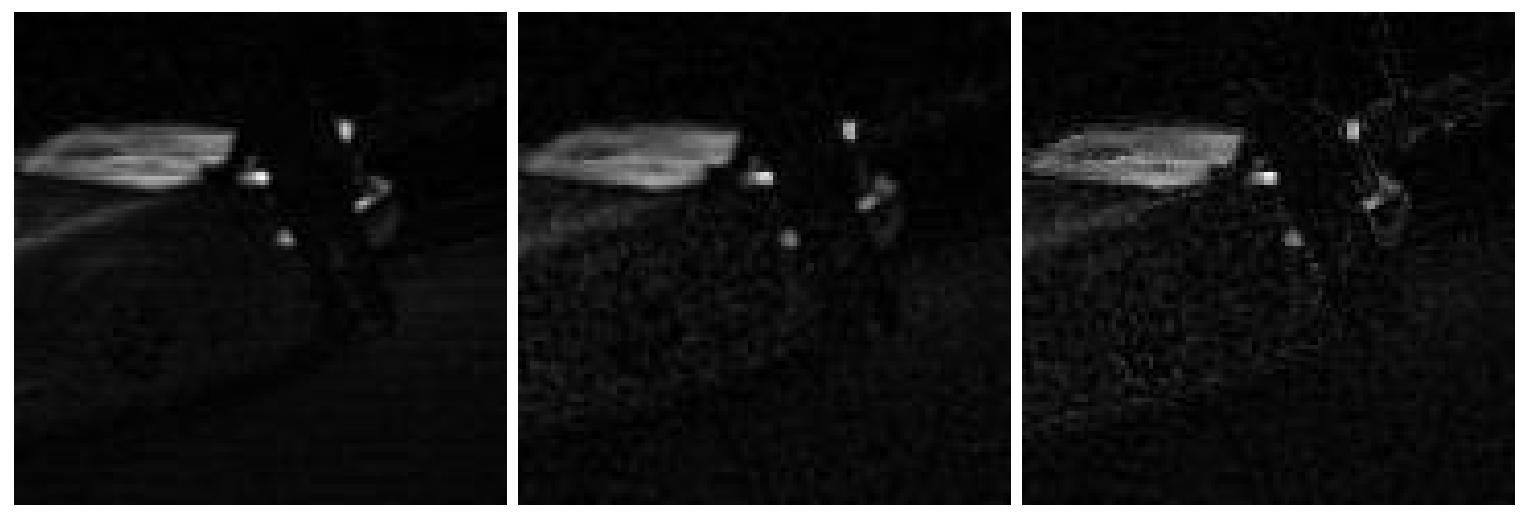

Figure 6. Estimates of DOLP. From left to right: Pristine, Proposed Method, Traditional Method 